\title{
Low online search interest in teleneurology before and during COVID-19 pandemic: an infodemiological study
}

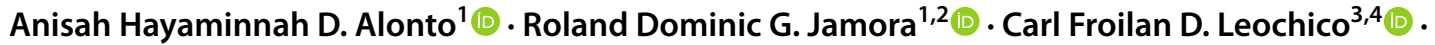 \\ Adrian I. Espiritu ${ }^{1,5,6}$ (D)
}

Received: 10 December 2021 / Accepted: 14 January 2022 / Published online: 24 January 2022

(c) Fondazione Società Italiana di Neurologia 2022

\begin{abstract}
Background The conduct of patient consults greatly changed during the time of the COVID-19 pandemic. There was a decrease in face-to-face patient consults and enhanced utilization of virtual consults. Infodemiological studies, using Google Trends, focus on internet search trends that may reflect public interest and awareness in diseases and as a proxy of public health risk perception.

Objectives We aimed to investigate the online behavior of internet users on teleneurology, telehealth, and telemedicine during the time of the pandemic, as reflected in search volume indices (SVI) projected in Google Trends.

Methods We used the data from Google Trends to quantify the interest of internet users in teleneurology, telehealth, and telemedicine. These keywords were entered in Google Trends as search terms. Data included were searches conducted from 2016 to 2020 to depict the transition into the pandemic.

Results The SVI of teleneurology was unchanged during the 5-year period search relative to the SVIs of telehealth and telemedicine. Contrary to the noted worldwide increase in the SVI of telehealth and telemedicine during the year of pandemic, teleneurology's SVI remained stable despite the increase of its utilization. Focusing on teleneurology, the highest SVI was observed in 2018.

Conclusion There was an increase in the SVI of telehealth and telemedicine, possibly implying an increase in the general awareness of these virtual methods of health care, as catalyzed by the pandemic. However, the stable SVI of teleneurology may signify that the public awareness regarding it remained unchanged despite the increase in application in clinics and hospitals.
\end{abstract}

Keywords Teleneurology $\cdot$ Telehealth $\cdot$ Telemedicine $\cdot$ Infodemiology $\cdot$ Google trends

Adrian I. Espiritu

aiespiritu@up.edu.ph

Anisah Hayaminnah D. Alonto

adalonto@up.edu.ph

Roland Dominic G. Jamora

rgjamora@up.edu.ph

Carl Froilan D. Leochico

cdleochico@up.edu.ph

1 Division of Adult Neurology, Department of Neurosciences, College of Medicine and Philippine General Hospital, University of the Philippines Manila, Manila, Philippines

2 Section of Neurology, Institute for Neurosciences, St. Luke's Medical Center, Quezon City and Global City, Philippines
3 Department of Rehabilitation Medicine, College of Medicine and Philippine General Hospital, University of the Philippines Manila, Manila, Philippines

4 Department of Physical Medicine and Rehabilitation, St. Luke's Medical Center, Quezon City and Global City, Philippines

5 Department of Clinical Epidemiology, College of Medicine, University of the Philippines Manila, Manila, Philippines

6 Division of Neurology, Department of Medicine, St. Michael's Hospital, University of Toronto, Toronto, Canada 


\section{Introduction}

Teleneurology is the practice of delivering neurologic care to distant areas through the use of modern communication technology via videoconferencing or email to enable neurology to be practiced when the neurologist and patient are not present in the same place [1]. Teleneurology narrows the gap between patients with neurological disease and neurologists, especially in areas without a specialist [1]. However, its adoption has been slow. In the recent need for social distancing during the coronavirus disease 2019 (COVID-19) pandemic, the utilization of teleneurology has significantly increased [2, 3]. Even in areas where there are neurologists, teleconsultations are advocated over in-person consultations for non-emergency cases to minimize unnecessary COVID19 risks [2, 3]. The COVID-19 pandemic prompted changes in the healthcare system to ensure continuity of care for patients with chronic neurological diseases such as neuromuscular [4-6], neurodegenerative [7], and neurovascular conditions [8]. Teleneurology may detect early symptoms and signs of disease worsening, prevent life-threatening complications, and notice changes in the clinical condition that would require further evaluation in a hospital setting [5], saving patients from unnecessary possible COVID-19 exposure in the clinics and hospitals.

As a result of the increase in the utilization of teleneurology in clinical practice, the general public, particularly internet users, may have increased awareness and online search activity about teleneurology, as may be reflected in the SVIs of Google Trends.

Google Trends, a source of reversed engineered data, provides access to internet search patterns by analyzing web queries on the Google search website and other affiliated Google sites [9]. The data are normalized in terms of search frequency and presented in relative search volumes [10]. The website provides both real-time and archived information on Google queries from 2004 to the present [11]. The use of Google Trends in research studies began in 2009 when it was demonstrated that Google Trends traced and predicted the spread of influenza earlier than the Centers for Disease Control and Prevention [12]. Currently, Google Trends is one of the most popular online tools in addressing health issues and topics by looking at internet search trends that may reflect public interest and awareness [11].

In this study, we aimed to describe the worldwide search interest about teleneurology among internet users before and during the pandemic, including online search behaviors related to terms like telemedicine and telehealth, using Google Trends.

\section{Methods}

This study investigated search trends of teleneurology and its related terms to look into the online interest of internet users, using SVI in Google Trends, which may be reflective of public awareness regarding the said concepts. A search volume index, available on the Google Trends website, reflects an estimate of search activity on a particular topic relative to the highest volume of searches documented within a specific period and geographic location. Hence, SVIs do not necessarily report the absolute volume of searches for a specific term [13]. Instead, the data are normalized and expressed on a scale of 0 to 100 , wherein a higher score reflects greater interest for a particular search term [10]. SVIs have been previously used to evaluate population interest in health-related topics [5, $8,9]$.

The keywords teleneurology, telemedicine, and telehealth were entered in Google Trends as search terms and were explored under the health category in Web Search worldwide. As teleneurology is a subset of telemedicine, which is often interchanged with telehealth, all these terms were included. However, telehealth and telemedicine have distinct definitions. Telemedicine is a clinical service that seeks to improve a patient's health remotely by using twoway, real-time, interactive communication between the patient and the physician [10], while telehealth encompasses health assessment, diagnosis, intervention, consultation, supervision, and information delivery over a distance [14]. Hence, telehealth is considered a broader concept of telemedicine [14]. The search period was set from January 1, 2016, to December 31, 2020, to cover the possible change in online search trends from the few years prior to and during the pandemic. A separate search for teleneurology using the same aforementioned parameters was done to further characterize the internet users' search behavior for teleneurology.

\section{Results}

Among the three related terms searched in this study, telehealth had the highest SVI in 2020, coinciding with the COVID-19 pandemic (Fig. 1). It was most searched in Canada and Australia (Table 1). Meanwhile, telemedicine ranked second worldwide, with the highest SVIs coming from the United States, Bangladesh, and the Philippines (Table 1). Telemedicine and telehealth had SVIs ranging from 2 to 7 before the pandemic (i.e., 2016-2019), and suddenly peaking in March 2020, when the WHO officially declared COVID-19 as a global health emergency 
Fig. 1 Google Trends graph depicting online search trends for teleneurology, telemedicine, and telehealth worldwide from 2016 to 2020

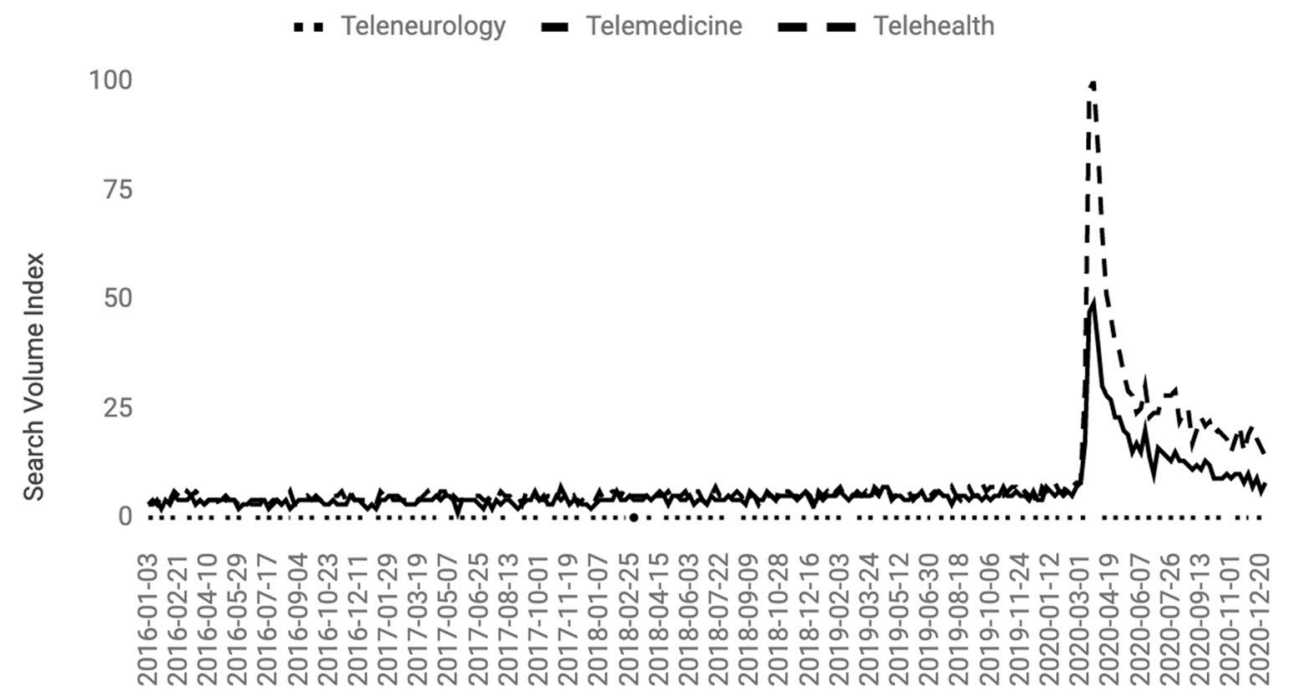

Week

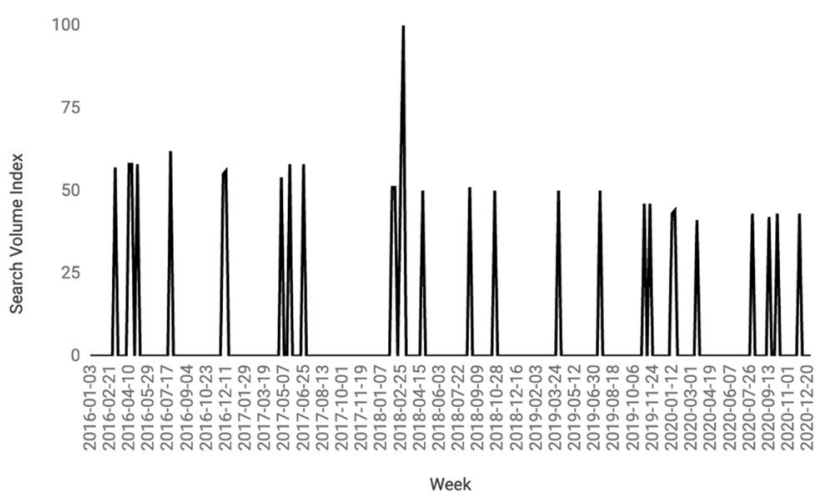

Fig. 2 Google trends graph depicting online search trends for teleneurology worldwide from 2016 to 2020

\section{Discussion}

This study showed that there was a worldwide increase in the SVI for the keyword's telemedicine and telehealth at the start of the pandemic in 2020 . This might possibly signify an increase in the interest in these terms. However, relative to the SVIs of telemedicine and telehealth, teleneurology's SVI remained unchanged for the 5-year period search (Fig. 1). In fact, when a search was done for teleneurology alone, the peak SVI was noted pre-pandemic, particularly in 2018 (Fig. 2), which was coincidentally the year when a US-based national provider of acute telemedicine technology created a teleneurology council for the advancement of remote neurology care [16]. Therefore, despite the increase in the utilization of teleneurology among neurologists during the period of the pandemic, the search behavior of internet users regarding teleneurology remained unchanged. 
Telemedicine and telehealth had higher SVIs compared to teleneurology, likely because these were broader terms that is encompassing many fields of medical specialization, as compared to teleneurology, which is a more specialized service specific to patients with neurologic problems [17]. The increase in the utilization of teleneurology during the time of pandemic would have possibly increased the awareness of the general public regarding the use of technology to connect with their neurologists and other doctors. However, in Fig. 2, we saw that the peak of teleneurology SVI was during pre-pandemic. This was probably because end users did not particularly use the exact word as their search term in Google and would have rather used the broader key concepts. The concept of teleneurology is likely more known among neurologists, comprising a relatively small percentage of the population of physicians worldwide [18]. Therefore, teleneurology is recommended to be promoted more as it may improve the accessibility of specialized care for patients [19]. This kind of virtual consult is of great aid for patients with limited mobility and those living far from the hospitals where specialists practice [20]. This can also be promoted to other medical doctors, such as general practitioners practicing in rural areas where there are very few specialists to guide them on patient management [21]. This can be an effective tool for reducing the treatment gap and has the potential to serve geographically isolated and disadvantaged areas. Due to the increase in the utilization of technology for neurologic consults, there is also a need for more research about evaluating teleneurology services to intensify training workshops for neurologists and improve facilities to appropriately serve more patients in need [20].

Even before the pandemic, teleneurology was shown to provide great benefit to populations with limited access to general and specialized neurological care, such as rural populations and those patients with limited mobility [22]. Telestroke or telemedicine for stroke was utilized as early as 1999 when there was a need to enhance and expedite acute stroke care in local communities without neurologists [18]. But its adoption has been slow because of laws, regulations, and policies [23]. However, in the study by McGinley, they showed that the overall teleneurology visit volume during March 2020 increased by 533\% in Neurological Institute at Cleveland Clinic [2]. This increase in the utilization of technology for outpatient consults was brought about by the movement restrictions caused by the pandemic and was encouraged by policymakers, regulators, and payers by loosening policy restrictions [23]. The pandemic might have accelerated the adoption of teleneurology worldwide as it has changed some laws and policies regarding reimbursements and regulations, which were among the factors that had prevented its emergence in the healthcare landscape [2]. This documented increase in the virtual consults among patients with the neurological condition did not, however, reflect as an increase in the online search activity or SVI of teleneurology, for which a high SVI may possibly mirror an increase in awareness of the general public regarding it.

Teleneurology was most searched in the US, followed by India. The increased SVI from the US was likely because teleneurology was more practiced and utilized in a high-income country with an accessible internet connection for the public. In high-income countries with a high-speed internet connection, it would be less complicated to conduct a virtual consult than in areas with a very poor internet connection, which might affect communication between end-users. This convenient and uninterrupted virtual consultation between neurologist and patient, which made specialized care more accessible, might have made teleneurology more known in high-income countries. In India, a low-resource setting, however, they had a government-sponsored Indian program that provided teleneurology, telepsychiatry, teleneurorehabilitation, teleradiology, and tele-Ayurveda [20]. These services had been available in India since 2010, allowing neurologists to provide remote service to indigent patients and support primary care doctors in managing common neurological disorders [20]. The Indian government and private sector worked together to provide high-speed internet for centers to make the services accessible [20].

The presence of a high-speed internet connection makes it more convenient to facilitate other virtual services, on top of the virtual consults, such as the delivery of health information and health systems management, as this allows stakeholders to effectively use technology for the said online services [24]. Telehealth, which is a more encompassing concept involving not only online consultation but also other health care services, is more famous in high-income countries with high-speed internet connections such as Canada, Australia, New Zealand, US, and Singapore (Table 1). Telemedicine, however, which is a narrower concept involving only the provision of clinical services such as follow-up visits, is more searched in low to middle-income countries such as Bangladesh, the Philippines, and Ghana. Technical factors such as slow internet speed and limited internet coverage are some of the common challenges faced by low to middleincome countries when utilizing health care advances such as the use of technology for healthcare delivery [25].

This study has some inherent limitations. The SVI from Google Trends, a reflection of the online search behavior of internet users, requires access to the internet and the use of Google search engine and its associated sites. Hence, people without internet access and internet users who use different search engines were not taken into consideration. The use of SVI from Google Trends can only be a surrogate marker for population interest for teleneurology. It is still unclear whether changes in online search behavior or activity translate to changes in awareness of the general public [26]. However, since there are more than 3 billion internet 
users around the world and Google is the most widely used search engine [13], Google Trends may still be a useful tool to estimate public interest.

In this study, we have shown that there was an increase in the Google Trends SVI of telehealth and telemedicine during the period of the pandemic. This may translate as an increase in awareness among internet users regarding these healthcare-providing services. Teleneurology, however, did not show a change in SVI despite the increase in its utilization during the time of the pandemic. This unchanged search behavior could possibly signify that this had not changed the awareness of the general public regarding the possible use of technology to have virtual consults with neurologists. Therefore, teleneurology is recommended to be promoted more to neurologic patients, especially those with stable neurologic conditions that need monitoring and/or adjustment of maintenance medications such as patients with epilepsy, Parkinson's disease, dementia, and others. Even after this COVID-19 pandemic, the use of these virtual consults will help them make specialized neurologic care more available to them. In line with the increase in the utilization of these services, neurologists should also be more trained in conducting these virtual consults to improve the quality of these services. These virtual consults may also be of great help not just to patients but also to general practitioners who are practicing in rural areas where specialists are few. Through teleneurology, neurologists may guide them in the management of possible neurologic patients. In conjunction with awareness campaigns regarding teleneurology and workforce training, there must also be an improvement in the technical factors involved in the conduction of these services, such as internet speed and coverage, equipment, and secure software to store data [27]. These technical factors mostly concerned those in the low to middle-income countries who continue to face these barriers to the emergence of such health care services [25].

\section{Conclusion}

The increase in SVI of telehealth and telemedicine may signify an increase in awareness among the general public. This has been likely due to the significant increase in the utilization of these services due to the movement restrictions brought about by the pandemic. There is also an increase in the application of teleneurology in clinics and hospitals; however, this did not reflect as an increase in the online search activity of teleneurology, which may signify that there has been no change in public awareness regarding it. Therefore, the use of teleneurology is encouraged, and more research is suggested to be conducted regarding the quality of services it can offer. As teleneurology may continue to be beneficial even after the pandemic, training and workshops are advocated among neurologists on how to use different platforms appropriately to provide quality service to patients. Along with awareness campaigns and workforce training, technical factors, such as internet coverage and speed, are also suggested to have improvements to make teleneurology more accessible and available to patients with neurologic conditions.

Author contribution AHDA: conceptualization, data curation, formal analysis, interpretation of data, writing-original draft, writing-review, and editing. RDGJ: conceptualization, data curation, formal analysis, interpretation of data, writing-original draft, writing-review, and editing. CFDL: conceptualization, data curation, formal analysis, interpretation of data, writing-original draft, writing-review, and editing. AIE: conceptualization, data curation, formal analysis, interpretation of data, writing-original draft, writing-review, and editing.

\section{Declarations}

Ethics approval There was no identifying information on individual persons in this infodemiological study. Thus, ethical board review was not deemed necessary for this study.

Conflict of interest The authors declare no competing interests.

\section{References}

1. Patterson V (2005) Teleneurology. J. Telemed. Telecare. SAGE PublicationsSage UK: London, England; [cited 2021 Feb 14]. p. 55-59. Available from: http://journals.sagepub.com/doi/10.1258/ 1357633053499840

2. McGinley MP, Ontaneda D, Wang Z et al (2020) Teleneurology as a solution for outpatient care during the COVID-19 pandemic. Telemed e-Health 26:1537-1539

3. Roy B, Nowak RJ, Roda R et al (2020) Since January 2020 Elsevier has created a COVID-19 resource centre with free information in English and Mandarin on the novel coronavirus COVID19. The COVID-19 resource centre is hosted on Elsevier connect, the company's public news and information.

4. Bombaci A, Abbadessa G, Trojsi F et al (2020) Telemedicine for management of patients with amyotrophic lateral sclerosis through COVID-19 tail. 20-24

5. Ricciardi D, Casagrande S, Iodice F et al (2021) Myasthenia gravis and telemedicine: a lesson from COVID-19 pandemic. Neurol Sci 42:4889-4892. Available from: https://doi.org/10. 1007/s10072-021-05566-8

6. Spina E, Trojsi F, Tozza S et al (2021) How to manage with telemedicine people with neuromuscular diseases? Neurol Sci 42:3553-3559

7. Elbeddini A, To A, Tayefehchamani Y et al (2020) Potential impact and challenges associated with Parkinson's disease patient care amidst the COVID-19 global pandemic. J Clin Mov Disord $7: 1-7$

8. Iodice F, Romoli M, Giometto B et al (2021) Stroke and digital technology: a wake-up call from COVID-19 pandemic. Neurol Sci 42:805-809

9. Nuti SV, Wayda B, Ranasinghe I et al (2014) The use of google trends in health care research: a systematic review. PLoS One 9 
10. Strzelecki A, Rizun M (2020) Infodemiological study using google trends on coronavirus epidemic in Wuhan, China. Int J online Biomed Eng 16:139-146

11. Mavragani A, Ochoa G (2019) Google Trends in infodemiology and infoveillance: methodology framework. JMIR Public Heal Surveill 5:e13439

12. Ginsberg J, Mohebbi MH, Patel RS et al (2009) Detecting influenza epidemics using search engine query data. Nature 457:1012-1014

13. Paguio JA, Yao JS, Dee EC (2020) Silver lining of COVID-19: heightened global interest in pneumococcal and influenza vaccines, an infodemiology study. Vaccine 38:5430-5435

14. Kichloo A, Albosta M, Dettloff K et al (2020) Telemedicine, the current COVID-19 pandemic and the future: a narrative review and perspectives moving forward in the USA. Fam Med Commun Health $8: 1-9$

15. Timeline of WHO's response to COVID-19. [cited 2021 May 20]. Available from: https://www.who.int/emergencies/diseases/ novel-coronavirus-2019/interactive-timeline?gclid=Cj0KCQjw7p KFBhDUARIsAFUoMDaNB7wVqDf14g48KVEv3TQ1vP7lfwQ NDa1zNDGftk6LZpR2grtMsO4aAu3TEALw_wcB\#!

16. SOC Telemed Creates teleNeurology Council. [cited 2021 May 17]. Available from: https://www.prnewswire.com/news-releases/ soc-telemed-creates-teleneurology-council-300764203.html

17. Leochico CFD, Espiritu AI. Global online interest in telehealth, telemedicine, telerehabilitation, and related search terms amid the COVID-19 pandemic: an infodemiological study.

18. Racette BA, Holtzman DM (2014) Supply and demand analysis of the current and future us neurology workforce. Neurology 82:2254-2255

19. Ross L, Bena J, Bermel R et al (2021) Implementation and Patient experience of outpatient teleneurology. Telemed e-Health $27: 323-329$
20. Gowda G, Manjunatha N, Kulkarni K et al (2020) A collaborative tele-neurology outpatient consulation service in Karnataka: seven years of experience from a tele-medicine center. Neurol India. [cited 2021 Apr 21];68:358. Available from: http://www.neuro logyindia.com/text.asp?2020/68/2/358/280644

21. Almallouhi E, Al Kasab S, Harvey JB et al (2020) Teleneurology network to improve access to neurologists for patients in rural areas: a real-world experience. Telemed e-Health 26:110-113

22. Patel UK, Malik P, DeMasi M et al (2019) Multidisciplinary approach and outcomes of tele-neurology: a review. Cureus 11:1-15

23. Klein BC, Busis NA (2020) COVID-19 is catalyzing the adoption of teleneurology. Neurology 94:903-904

24. Bauerly BC, McCord RF, Hulkower R et al (2019) Broadband access as a public health issue: the role of law in expanding broadband access and connecting underserved communities for better health outcomes. J Law, Med Ethics 47:39-42

25. Leochico CFD, Espiritu AI, Ignacio SD et al (2020) Challenges to the emergence of telerehabilitation in a developing country: a systematic review. Front Neurol 11

26. Rovetta A (2021) Reliability of Google Trends: analysis of the limits and potential of web infoveillance during COVID-19 pandemic and for future research. Front Res Metrics Anal 6

27. Moccia M, Brigo F, Tedeschi G et al (2018) Neurology and the Internet: a review. Neurol Sci 39:981-987

Publisher's note Springer Nature remains neutral with regard to jurisdictional claims in published maps and institutional affiliations. 